\title{
Probabilistic energy storage sizing for reducing wind power forecast uncertainty
}

\author{
H. Bludszuweit ${ }^{1}$ and J.A. Domínguez ${ }^{2}$ \\ ${ }^{1}$ Electrical Engineering Division \\ CIRCE Foundation \\ C / María de Luna 3, 50018 Zaragoza (Spain) \\ Phone number: +34 97676 2404, e-mail: hblud@unizar.es \\ ${ }^{2}$ Department of Electrical Engineering \\ University of Zaragoza \\ C / María de Luna 3, 50018 Zaragoza (Spain) \\ Phone number: +34 97676 2401, e-mail: jadona@unizar.es
}

\begin{abstract}
A novel method is proposed for designing an energy storage system (ESS) which is dedicated to reducing the uncertainty of the short term wind power forecast. The investigation focuses on the statistical behaviour of the forecast error and the state of charge (SOC) of the ESS. This approach gives an insight into the influence of the forecast conditions on the distribution of SOC. With this knowledge, an optimised sizing of the ESS can be done with a well defined uncertainty limit

One-year power output data measurements and two types for forecast were used for this study. In addition, different forecast quality degrees are simulated based on the persistence approach. With the forecast data, empirical probability density functions (pdf's) of the SOC are generated which is the base of the proposed method.

This approach can lead to a considerable reduction of the ESS and provides important information about the unserved energy. This unserved energy is the remaining forecast error or uncertainty. As a consequence, the proposed probabilistic method permits the sizing of the energy storage system as a function of the desired remaining forecast uncertainty.
\end{abstract}

\section{Key words}

wind power, forecast error, energy storage sizing, probability density function.

\section{Introduction}

In recent years wind energy has grown to an extent that its impact on the electric power system becomes relevant in many regions [1]-[3]. On the other side, generation costs are falling and the participation in short term energy markets becomes interesting [4]-[7]. For both cases wind power forecasts are crucial. The power system operators make reserve allocation plans taking into account wind power predictions and in day-ahead energy markets the benefit depends on the forecast accuracy. Forecast systems are improving steadily but it will remain always an uncertainty. Considering the importance of wind power forecasts, it becomes clear that the reduction of the forecast error is needed for a further development of wind power.

There are very few publications until now on probabilistic ESS sizing. The usefulness of ESS in electricity markets due to wind power output smoothing and reduction of forecast errors is shown in [8]. But here, only 9 possible ESS sizes are taken into account (combinations of 3 power and 3 energy capacities). Thus, the actual sizing is based on simple try and error. A similar approach is proposed in [9].

An interesting approach is proposed by Barton and Infield [10], which is based on spectral analysis of wind and solar resources combined with daily load profiles. The model is applied to a stand-alone system where the storage is calculated for different levels of mean load. The ESS is designed for a 24-h time horizon and a worst case scenario ("worst day of the year") is used to obtain the ESS size and unserved energy. A drawback of the method described in [10] is that there are some assumptions like probability distributions of net power to and from the store, which are not further specified.

In [11] and [12] the possible smoothing effect of an ESS is simulated with an exponential moving average (EMA), which is the same as a first order low pass filter. This kind of smoothing with ESS is very similar to the persistence approach, used in this paper. to simulate forecasts. For simplicity, a simple mean as forecast is preferred here as a reference.

In this study a novel method is proposed for designing an energy storage system (ESS) to reduce the forecast uncertainty. As point of departure, an ESS can be defined which compensates $100 \%$ of the uncertainty. This means it absorbs any deviation from the forecast and will be called here the 'zero error case'.

Unfortunately, the energy capacity of such a system would get quickly very large with growing forecast horizon. It will be shown that if a residual error or uncertainty is permitted, power and energy capacity of the ESS can be reduced drastically. 
The presented method quantifies the remaining uncertainty as a function of the reduction of the ESS size (power and energy capacity) compared to the zero error case. To achieve this, at first the statistical patterns of forecast errors and the stage of charge (SOC) of the ESS are studied for the zero error case. In a second step power and energy capacities are reduced and the resulting uncertainty is estimated.

As a summary, the presented method estimates the energy amount which is not compensated by the ESS if its size is reduced. This can be very useful to find the pay-off between income losses due to forecast errors and investment costs for the ESS.

Data from these two forecast methods will be examined in this paper and termed as MSE and MCC. These two real world scenarios are compared with results from the persistence model, proposed by the authors in [13].

\section{Forecast data}

In this paragraph, the forecast data used for this study is briefly described. Real world forecast data is compared with simulated forecasts based on the persistence approach.

\section{A. Real world forecast data}

In the present work, one-year time series of two different wind power forecasts for a wind farm in Portugal are analysed. Forecasts from 7 to $72 \mathrm{~h}$ ahead are given in 30min time steps for an entire year, being updated every 24 hours. In this study, only the 24 to 48 -h ahead forecast is used.

Forecasts for this site are available from two different models, which are described more in detail in [14]. According to [14], in an adaptive forecast system classical Mean Squared Error (MSE) criterion and a Maximum Correntropy Criterion (MCC) are used as cost function to train a neural network $(\mathrm{NN})$.

\section{B. Online bias-correction}

In this case study, bias correction was needed for realworld data in order to obtain bias-free data. State of the art forecast models include model output statistics (MOS) for this purpose.

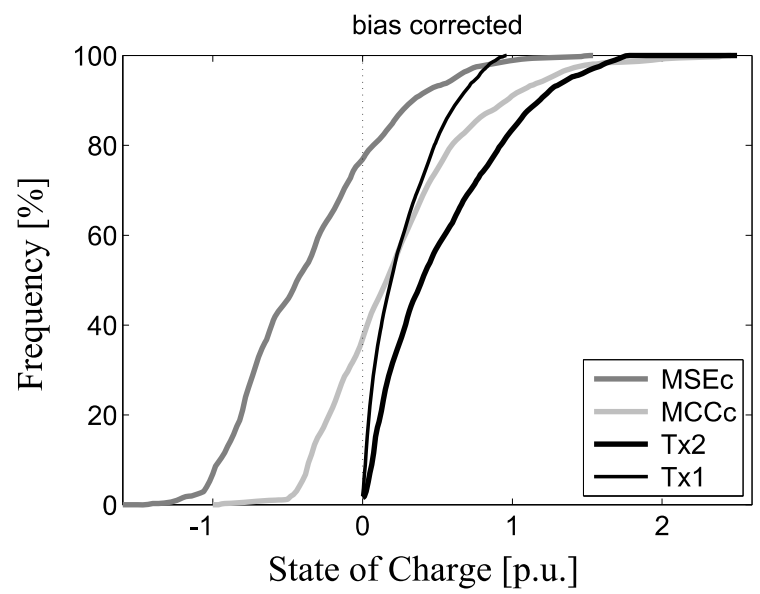

In this paper, a very simple method is proposed for online-correction of seasonal bias. An exponential moving average is applied on observations of the past 7 days. It can be shown, that for averaging windows of approximately 7 days, a payoff is obtained between biascorrection and forecast quality [15].

All real-world datasets are tested on seasonal bias and if necessary, are corrected with the averaging method described above. A good method to evaluate the effect of bias-correction of the forecast data is the representation of the cumulated forecast error. This in turn can be interpreted as the state of charge (SOC) of an ESS which is compensating this error. Such a representation is shown in Fig. 1, for the data investigated here. It can be observed that the cumulative histograms (cdf) of SOC for bias-corrected data is much more similar to the persistence forecasts $\mathrm{Tx} 1$ and $\mathrm{Tx} 2$. Raw forecast data show large deviations in SOC which indicates a strong bias in the forecast error.

\section{Storage sizing methodology}

In this paragraph, a probabilistic methodology is presented which permits the estimation of ESS size as a function of unserved energy. The ESS is designed to compensate forecast errors up to a certain extent. The level of compensation is expressed by the integral of uncompensated forecast errors, termed unserved energy $E_{u}$. Hence, the unserved energy is defined as the energy that cannot be absorbed or supplied by the ESS. This value is adopted here as sizing criterion. If $E_{u}$ is expressed as a percentage of total generated wind energy $E_{\text {total }}$ in the observed time interval (e.g. one year), it may be defined by

$$
e_{u}=100 \% \cdot \frac{E_{u}[\mathrm{kWh}]}{E_{\text {total }}[\mathrm{kWh}]}
$$

where $E_{u}$ is the unserved energy by the ESS and $E_{\text {total }}$ the total generated wind energy.

Sizing of power and energy requirements is done separately and merged at the end by interpolation.

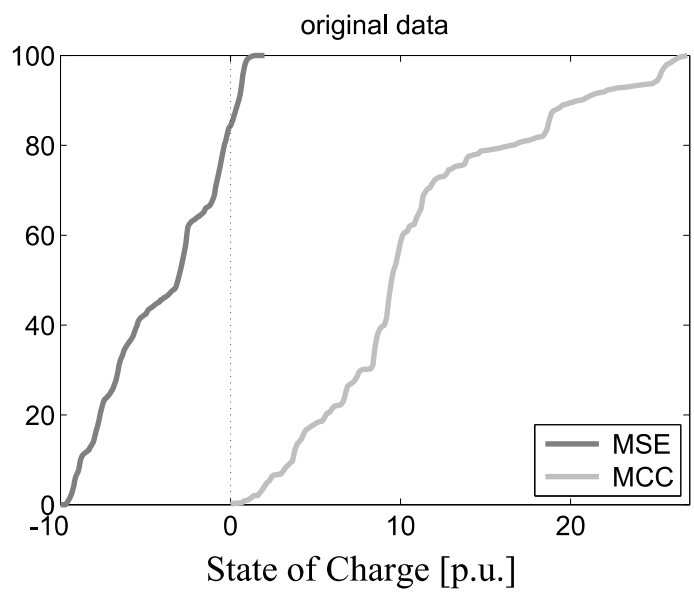

Fig. 1. Cumulative histograms (cdf) of SOC for different 24-h forecast scenarios, left: persistence forecasts Tx1 and Tx2 and bias corrected forecasts MSEc and MCCc, right: raw forecast data MSE and MCC. 


\section{A. Sizing of ESS power}

The rated power $P_{E S S}$ of an ESS defines its capacity to react quickly on events with very short time constants and is often decisive for the determination of the most appropriate ESS technology. In the following the normalised ESS power $p_{E S S}=P_{E S S} / P_{\text {inst }}$ will be used.

The first guess for $P_{E S S}$ is the installed wind power $P_{\text {inst }}$. Thus, $p_{E S S}=1$ can serve as a reference because it guarantees any power demand to be served.

As shown in [13], the unserved energy $e_{u p}$, due to reduced ESS power rating, can be obtained directly from the forecast error distribution. If $f(\varepsilon)$ is the probability density function (pdf) of the forecast error $\varepsilon$ and $p_{E S S}$ the normalised rated power of the ESS, $e_{u p}$ can be calculated as

$$
e_{u p}=\frac{1}{\bar{p}} \int_{p_{E S S}}^{1} f(\varepsilon) \cdot\left(\varepsilon-p_{E S S}\right) d \varepsilon
$$

Modern probabilistic forecast tools provide these distributions, but as shown in [13], the pdf can be estimated from relatively small datasets (one year for example) using the beta distribution. This method is referred as 'Betafit'. In this paper, estimated pdf are used and results are compared with time step simulations.

\section{B. Sizing of ESS energy capacity}

The definition of energy capacity requirements is more complex. Here a two-step method is proposed. The first step consists in the estimation of the energy throughput of the ESS. In a second step, saturation times of the ESS are estimated for the case that ESS energy capacity $E_{E S S}$ is reduced below the limit needed for $100 \%$ compensation of the forecast errors.

It will be shown that unserved energy $e_{u e}$ may be estimated in a first approach by

$$
e_{u e} \approx t_{s a t} \cdot N M A E
$$

where $t_{\text {sat }}[\%]$ is the saturation time and NMAE [p.u.] the normalised mean absolute error.

In the next paragraphs, saturation time $t_{\text {sat }}$ and energy throughput ratio ETR will be explained. While ETR in this case only depends on the forecast error, $t_{s a t}$ is obtained as a function of ESS energy capacity $E_{E S S}$.

In (3) $N M A E$ represents the energy throughput ratio of the ESS. Only in this special case of forecast error compensation the throughput is equal to the mean absolute error (MAE).

Thus, in a more general formulation the energy throughput ratio ETR would be used instead of $N M A E$, which can be defined as

$$
E T R=\frac{E_{t p}}{E_{\text {total }}}=\frac{\int\left|p_{s}(t)\right| d t}{\int p_{w}(t) d t}
$$

where $E_{t p}$ is the annual ESS energy throughput, $E_{\text {total }}$ the total generated wind energy and $p_{s}$ the normalised storage power, $p_{w}$ the normalised measured wind power.

In this case $E_{\text {total }}$ is the wind energy generation but it could represent any reference such as the difference between generation and consumption in case of demand forecast, for example.
Note that ETR is related to the number of equivalent full cycles of the ESS, if ESS energy capacity $E_{E S S}$ is known. Here ETR is preferred, since it describes a property of the energy input variable (in this case forecast error $\varepsilon$ ) and is independent of the ESS size or technology.

The value of $E_{t p}$ is the integral of absolute energy input and output of the ESS, which means that charged and discharged energy is summed. For an ideal ESS with $100 \%$ storage efficiency the input and output energy is equal, as the energy balance has to be maintained always. Because this idealised case is of special interest here, the ideal throughput ratio $E T R_{0}$ is introduced here. In this section, only $E T R_{0}$ is considered for sizing.

To obtain the throughput ratio, assuming an ESS efficiency $\eta$, it can be shown that the following equation describes the relationship between ideal and real throughput ratios [15].

$$
E T R=\operatorname{ETR}_{0} \frac{\eta+1}{2 \eta}
$$

The saturation time $t_{\text {sat }}$ can be estimated from the cdf of SOC, because the cumulative frequency $F_{s o c}$ of any value of SOC represents the percentage of time, the state of charge will be below this value. If ESS size is reduced to a value $e_{x}, 100-F_{s o c}\left(e_{x}\right)$ is the time, SOC will be greater than $e_{x}$ which is nothing else than $t_{s a t}$. As a first approximation, equation (10) can be formulated to estimate $t_{\text {sat }}$

$$
t_{\text {sat }}=100-F_{\text {SOC }}\left(e_{x}\right)
$$

where $F_{\text {soc }}$ is the cumulative distribution (or histogram) of SOC and $e_{x}$ the reduced ESS size.

An example how to obtain $t_{\text {sat }}$ is shown in Fig. 2 for the case of a Tx1 forecast of $12 \mathrm{~h}$. When the ESS size is reduced to $54 \%\left(e_{x}=0.54\right)$, a frequency value of $80 \%$ is obtained. This means that during $20 \%$ of all the operating time, SOC is above $e_{x}$ and the ESS will probably not serve the demand.

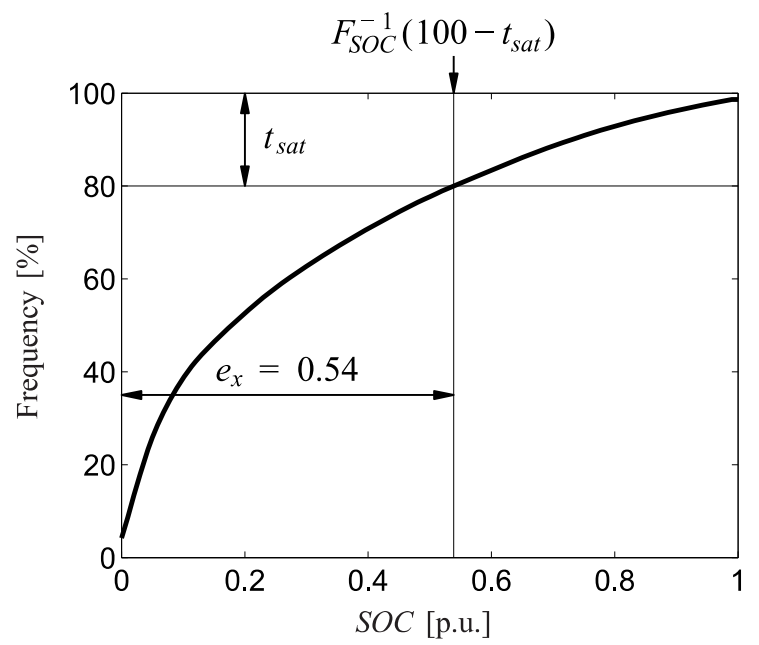

Fig. 2. Cumulative distribution (cdf) of SOC (Tx1, T = $12 \mathrm{~h})$ with representation of saturation time $t_{\text {sat }}$ for a reduced ESS size. 


\section{Simultaneous reduction of ESS power and energy}

In the preceding sections, unserved energy has been estimated separately for reduced ESS power and energy capacity. The estimation procedures were developed maintaining the rated power $P_{E S S}$ at 1 p.u. while $E_{E S S}$ was reduced or $E_{E S S}$ was maintained at the maximum value, while $P_{E S S}$ was reduced.

In order to estimate the effect of a simultaneous reduction of rated power and energy values, a discrete twodimensional interpolation algorithm is applied here. As a result, $e_{u}$ can be estimated for any combination of reduced power and energy capacity of the ESS.

Let $E T R_{0}$ be the throughput ratio of the ESS without any energy or power restrictions, i.e. all energy demand is covered by the ESS. Let further be $e_{u}$ the unserved energy due to any restrictions, then the reduced throughput ratio $E T R_{0}^{\prime}$ may be written as

$$
E T R_{0}^{\prime}=E T R_{0}-e_{u}
$$

where $E T R_{0}$ is the ideal throughput ratio if all errors are compensated and $e_{u}$ is the unserved energy.

Equation (7) is a general formulation without considering the contribution of ESS power and energy reduction to the global value of $e_{u}$. In order to split $e_{u}$ into its components $e_{u p}$ and $e_{u e}$ it is convenient to normalise $e_{u}$ by $E T R_{0}$. In (8) a 2D-interpolation algorithm is formulated for the case that relationships of $e_{u p}\left(P_{E S S}\right)$ and $e_{u e}\left(E_{E S S}\right)$ are given in discrete form.

$$
\operatorname{ETR}_{0, j k}^{\prime}=\operatorname{ETR}_{0}\left(1-\frac{e_{u p, j}}{E T R_{0}}\right)\left(1-\frac{e_{u e, k}}{E T R_{0}}\right)
$$

where $j$ is the index of the unserved energy vector $e_{u p}\left(P_{E S S}\right)$ and $k$ is the index of vector $e_{u e}\left(E_{E S S}\right)$.

If now $E T R_{0}^{\prime}$ is normalised with $E T R_{0}$, a general formulation for the calculation of $e_{u}$ may be

$$
e_{u}\left(P_{E S S}, E_{E S S}\right)=\operatorname{ETR}_{0}\left(1-e t r_{0}^{\prime}\right)
$$

where $e_{u}$ is the interpolated unserved energy [\%], ETR the ideal energy throughput ratio and etr $r_{0}$ the normalised reduced energy throughput ratio.

\section{Results}

The methodology presented above has been applied in a case study with real world forecast data. This study pursues two aims. First it validates the thesis that forecast errors can be modelled with a simple persistence approach. Secondly the sizing method is demonstrated and results are compared for different input data.

\section{A. Results for ESS power}

In Fig. 3 it can be seen at a glance that the Tx 1 scenario produces very similar results compared to real forecasts (MCC and MCCc). If ESS power is calculated with Betafit pdf estimation from Tx1 (bold black line), very good results are obtained. The situation is similar for the MSE forecast. Best results are obtained with bias corrected data MSEc and MCCc.

In Fig. 3 it can also be seen that estimations using Betafit with corrected data ('betafit MCCc') are closer to the results obtained from the histogram of the data ('hist
MCCc'). This shows the importance of bias-correction for the approximation of the forecast error pdf with Betafit. MCC forecast data showed a quite strong bias. Hence bias-correction had a strong impact on the results. Again it should be mentioned that wind power forecast data in general should not be biased. Thus, the weakness of the Betafit method in this point is not that relevant. In case of MCC, bias correction reduces the estimated ESS power by approximately $10 \%$.

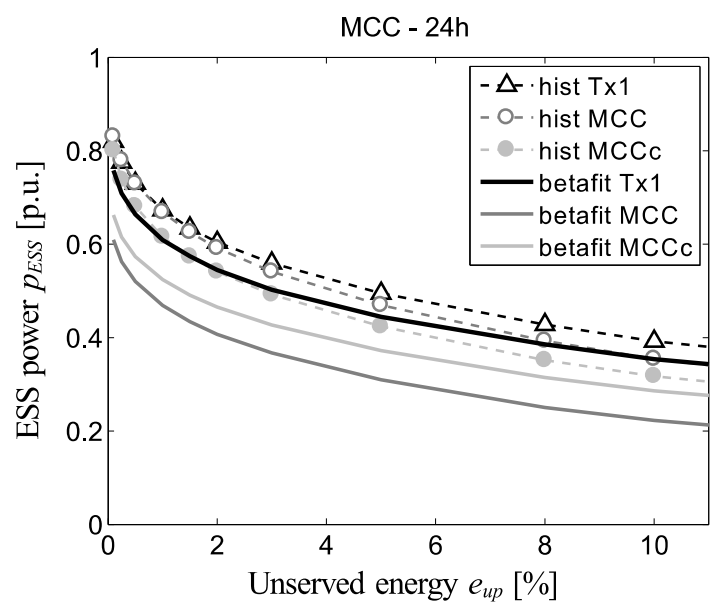

Fig. 3. Minimum ESS power as a function of unserved energy from real world 24-h forecast MCC compared with persistence scenario Tx1, calculated with observed histograms ('hist') and Betafit approximation ('betafit').

\section{B. Results for ESS energy capacity}

In Fig. 4 results are shown for sizing of ESS energy capacity. It shows bias corrected scenarios together with Tx1 and Tx2. Solid curves show estimations according to equation (3), dashed curves show results from time step simulations

It is seen that the Tx2 scenario can be used for ESS energy sizing only for values of unserved energy $e_{u e}>10 \%$. If lower values are desired, real world forecasts may demand significantly larger storage capacities. Scenario Tx1 is inadequate in this case.

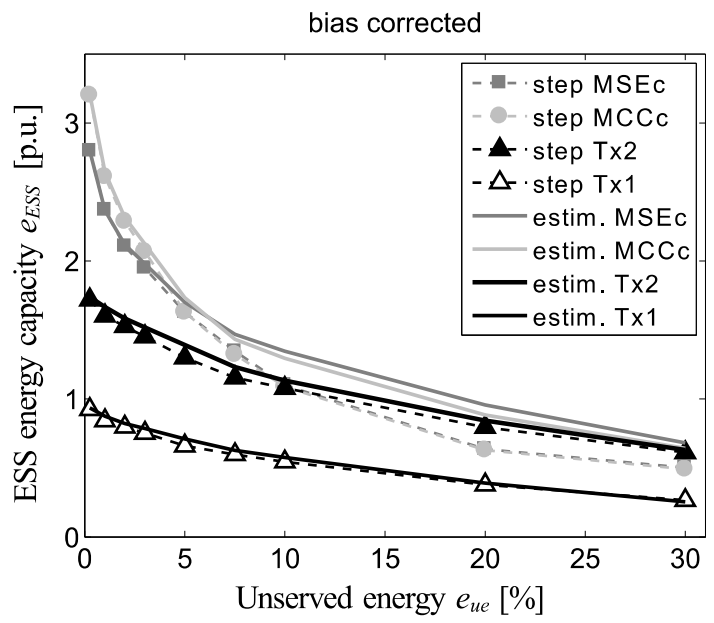

Fig. 4. Minimum ESS energy capacity as a function of unserved energy from dataset C for real world 24-h forecasts MSE and MCC compared with persistence scenarios Tx1 and Tx2, estimated from cdf of SOC ("estim.") and time step simulation ("step"). 


\section{Results for interpolation of ESS power and energy}

Interpolating the results, sizing surfaces can be obtained, giving an estimation of the unserved energy for every combination of reduced energy and power ratings. One example is given in Fig. 5. Here a reference system of $1 \mathrm{MW}$ and 24-h forecast is assumed, which represents $100 \%$ forecast error compensation in case of forecast scenario Tx1. Values of unserved energy are normalised by the maximum, which is equal to $E T R_{0}$ in this case.

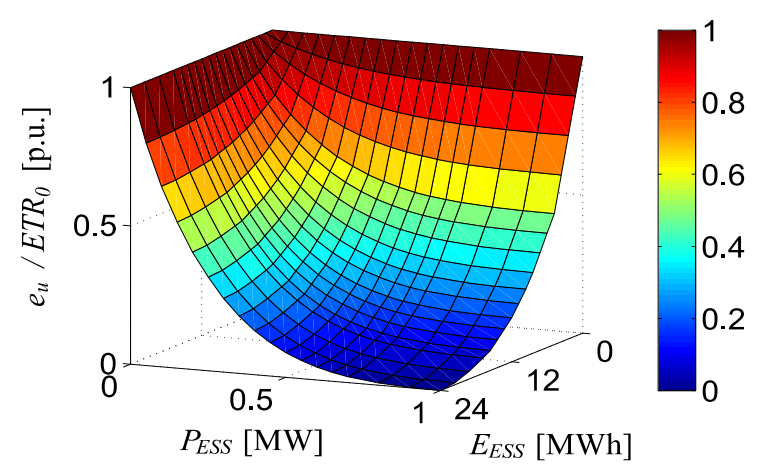

Fig. 5. 2D-interpolation of unserved energy, normalised with $E T R_{0}$ as a function of ESS size, reference case of 1 MW installed power and 24-h Tx1 forecast.

\section{Conclusions}

Five main conclusions can be derived from this study:

- Large ESS capacities are needed to reduce wind power forecast errors

- Permitting only small quantities of unserved energy, requirements for ESS are reduced drastically

- Persistence scenarios Tx1 (for power) and Tx2 (for energy) are valid for ESS sizing as they represent to a large extent statistics of real world forecasts

- The proposed sizing method performs well for 24-h forecasts with both, persistence and real world data

- Bias correction (MOS) improves model precision and reduces ESS capacity requirements

It could be demonstrated that forecast errors from the persistence scenarios can be used for ESS sizing. The sizing method is verified with real world forecast data. Especially for 24-h forecasts, the method presents relatively low errors which is interesting for day-ahead forecasts in electricity markets. Finally, model output statistics (MOS) such as the proposed online biascorrection revealed a great value for ESS sizing. Estimations are more precise and statistic parameters resemble more those from persistence scenarios.

From the case study it can be seen, that large capacities of energy storage are needed to compensate wind power forecast errors. On the other hand, only permitting amounts of unserved energy in the range of 5\% may reduce the required ESS size by up to $50 \%$.

\section{References}

[1] J. G. Slootweg, "Wind Power - Modelling and Impact on Power System Dynamics," Ph.D. dissertation, Technical University of Delft, Netherlands, 2003.

[2] J. O. G. Tande, "Exploitation of wind-energy resources in proximity to weak electric grids," Appl. Energ., vol. 65, pp. 395-401, 2000.

[3] T. Ackermann, K. Garner, and A. Gardiner, "Embedded wind generation in weak grids - economic optimisation and power quality simulation," Renew. Energ., vol. 18, pp. 205-221, 1999.

[4] P. Pinson, C. Chevallier, and G. Kariniotakis, "Trading Wind Generation From Short-Term Probabilistic Forecasts of Wind Power," IEEE T. Power Syst., vol. 22, no. 3, pp. 1148-1156, Aug. 2007.

[5] A. Fabbri, T. Gómez San Román, J. Rivier Abbad, and V. H. Méndez Quezada, "Assessment of the cost associated with wind generation prediction errors in a liberalized electricity market," IEEE T. Power Syst., vol. 20, no. 3, pp. 1440-1446, Aug. 2005.

[6] P. Pinson, C. Chevalier, and G. Kariniotakis, "Optimizing Benefits from Wind Power Participation in Electricity Markets using Advanced Tools for Wind power Forecasting and Uncertainty Assessment," in Proc. of EWEC'04, London, Nov. 2004.

[7] G. N. Bathurst, J. Weatherill, and G. Strbac, "Trading wind generation in short term energy markets," IEEE T. Power Syst., vol. 17, no. 3, pp. 782-789, Aug. 2002.

[8] M. Korpaas, A. T. Holen, and R. Hildrum, "Operation and sizing of energy storage for wind power plants in a market system," Int. J. Electr. Power Energy Syst., vol. 25, no. 8, pp. 599-606, Oct. 2003.

[9] G. N. Bathurst and G. Strbac, "Value of combining energy storage and wind in short-term energy and balancing markets," Electr. Power Syst. Res., vol. 67, no. 1, pp. 1-8, Oct. 2003.

[10] J. P. Barton and D. G. Infield, "A probabilistic method for calculating the usefulness of a store with finite energy capacity for smoothing electricity generation from wind and solar power," J. Power Sources, vol. 162, no. 2, pp. 943-948, Nov. 2006.

[11] J. V. Paatero and P. D. Lund, "Effect of Energy Storage on Variations in Wind Power," Wind Energy, vol. 8, no. 4, pp. 421-441, 2005.

[12] G. Koeppel and M. Korpås, "Improving the network infeed accuracy of non-dispatchable generators with energy storage devices," Electr. Power Syst. Res., vol. 78, no. 12, pp. 2024-2036, Dec. 2008.

[13] H. Bludszuweit, J. A. Dominguez, and A. Llombart, "Statistical Analysis of Wind Power Forecast Error," IEEE T. Power Syst., vol. 23, no. 3, pp. 983-991, Aug. 2008.

[14] R. J. Bessa, V. Miranda, and J. Gama, "Entropy and Correntropy Against Minimum Square Error in Offline and Online Three-Day Ahead Wind Power Forecasting," IEEE T. Power Syst., vol. 24, no. 4, pp. 1657-1666, Nov. 2009.

[15] H. Bludszuweit, "Reduction of the uncertainty of wind power predictions using energy storage," Ph.D. dissertation, Universidad de Zaragoza, Zaragoza, Jul. 2009. [Online]. Available: http://teide.cps.unizar.es:8080/ pub/publicir.nsf/codigos/0583/\$FILE/cp0583.pdf 